\title{
A Routing Algorithm for Wireless Sensor Networks
}

\author{
Fei Du \\ Electronic Information Department \\ Soochow University \\ Suzhou, China
}

\begin{abstract}
This paper presents a data-aggregation algorithm in the environment of wireless sensor network based on maximum lifetime. In this algorithm, the whole network is constructed into a tree structure with sink being the root of the tree. In the process of data-aggregation tree structure, in order to enable the data transmission path is the shortest, selected the smallest deflection angle relative to the sink as the next hop node. By the same time the remaining energy of the nodes is taken into account and achieves load balancing, maximizes the network lifetime.
\end{abstract}

Keywords-Wireless sensor networks, data-aggregation, tree structure, maximum lifetime, minimum routing

\section{INTRODUCTION}

WSNs(wireless sensor networks) is composed of a large number of low-cost micro-sensor nodes which are deployed in the monitoring region. It is a multi-hop and selforganization network by the formation of a wireless communication, for the purpose of collaborating perceiving, collecting and processing the information of network coverage in the perception of objects, and sends it to observers[1]. Sensors, sensing object and the observer constitute three elements of the sensor network. Wireless sensor networks have a wide range of applications in fields of environment, earthquakes, out space exploring and many other fields.

Sensor nodes are kinds of tiny embedded devices. Its processing power, storage capacity, communication bandwidth and energy to carry are very limited, especially for power supply energy, usually not easy for replacement. So, how to effectively use the limited energy becomes an important problem in sensor network design. And reducing the amount of data transmission in the network is one of the effective means to reduce the energy consumption [2]. Because the adjacent sensor to monitor the same event obtained with similar data, so the redundant data transfer in a certain degree will cause excessive consumption of energy, shorten the whole network lifetime. To avoid this problem, the process of sensor network data collection requires the use of data fusion technology, that is integrated treating the data from multiple sensor nodes, then complete information can be obtained.

\author{
Jianping Lv \\ Electronic Information Department \\ Soochow University \\ Suzhou, China
}

\section{DATA-AGGREGATION IN WIRELESS SENSOR NETWORKS}

\section{A. Concept of data-aggregation}

In wireless sensor networks, data-aggregation is mainly used to manipulate information collected from sensor nodes within the network. In point of view of application, the information in wireless sensor networks can be divided into the original information and useful information, the original information has been processed to remove unwanted components to retain useful composition, so becomes the useful information. In wireless sensor networks, the process of converting a number of relatively simple information to a single process is known as wireless sensor network dataaggregation (or data fusion) [3].

\section{B. Remaining energy computing}

Supposing original energy of the node as Er, after a period of network operation, at time $\mathrm{T} 1$, node $\mathrm{i}$ has sent $\mathrm{n} 1$ messages, received $\mathrm{n} 2$ messages (at this time, the energy of node $i$ is not less than the threshold Ev, the unit of message to receive and send is Ai), the remaining energy of node $i$ at the time of T1 can be presented by formula (1) [4].

$$
E_{r}(i)=E_{r}(i)-\sum_{k=1}^{n 1} E_{t}\left(i, j_{k}\right)-\sum_{l=1}^{n 2} E_{r}\left(i, j_{l}\right), j_{k}, j_{l} \in N_{i}
$$

\section{The tree of data-aggregation}

In sensor networks, the data collection of nodes is completed by reversing tree from the scattered-by-hop sensor nodes to the node that detected data is needed, so a tree structure from the source node to the root node is formed with a sink as the root, and source node as a leaf, namely data-aggregation tree [5], as shown in Figure 1. Expressed in this article for convenience, the node sent reporting data is called detection node. The node is called internal which is neither a fusion tree leaf nor a root. Internal nodes in the tree should not only be responsible for the data transmitted from parent node to the next level of forwarding functions, but also should complete data integration function, which 


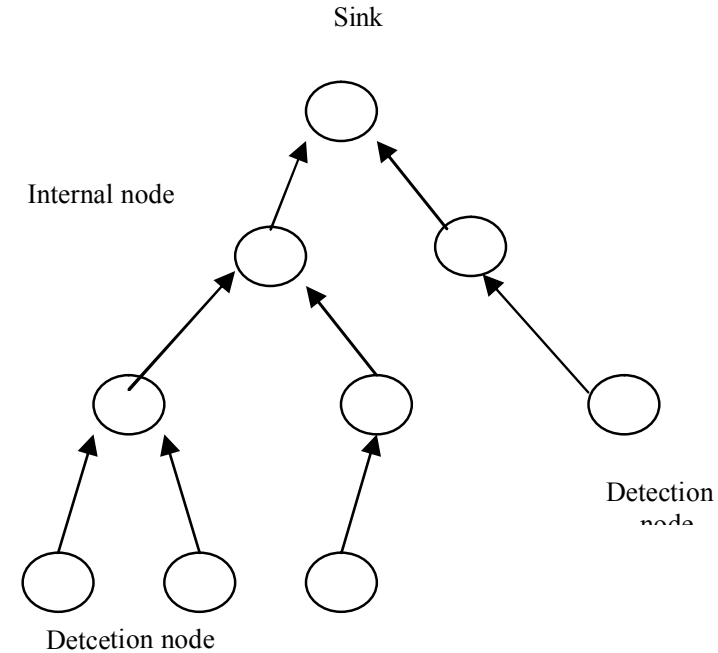

Figure 1 Tree structure of data-aggregation

is effectively integrating the data sent from the other nodes, eliminating redundant information and reducing data traffic.

Node in the tree structure (sink node excluded) can be divided into three classes: TYPE $=1$ : the nodes of simple detection which are leaf nodes in the integration of the tree structure. Leaf node is only detection node; TYPE $=2$ : fusion nodes and detection nodes. These nodes are not leaf node of the probe, but these nodes in the tree structure of data-aggregation should not only report the detection, but also transmit from other nodes on the data fusion processing and forwarding; TYPE = 3: simple integration nodes, this type of nodes in the tree structure are only responsible for data-aggregation and forwarding, that is, non-detection of the nodes [6].

\section{DATE-AGGREGATION ROUTING ALGORITHM BASED ON MAXIMUM LIFETIME}

In this paper, on analysis of characteristics of wireless sensor networks and wireless sensor network dataaggregation characteristics, we design a tree structure based on the maximum lifetine. In wireless sensor networks, energy consumption of sending information is related to transmission distance. Based on the direction of minimum ruoting, we choose the node which is the nearest to sink node as the next hop in order to achieve the shortest transmission path to save energy. The algorithm find its way from detection node initiated to begin selection of a layer of the father node, and so on, until all of the detection nodes are found in the path leading to the sink, forming a data fusion tree. In the algorithm, we suppose that the location of each node, aggregation node (sink node) location is known. Network sensor nodes are two-way communication between adjacent nodes periodically to change their information.

\section{A. The data structure of network nodes}

The information maintained by sensor node i includes: (1) Node level $\mathrm{Ci}$; (2) the father node Pi; (3)the list of child nodes $\mathrm{Ri}$; (4) the list of neighbor nodes $\mathrm{Ni}$; (5) the path list of detect node $\mathrm{Li}$; (6) node flag: flag $=0$ that the node has not been identified through other nodes and not the father of other nodes, not in the tree structure; flag $=1$ indicates that the section has been marked by other nodes, and is already another node's father in the fusion tree; (7) node type information TYPE; (8) node remaining energy Ei.

Each node in the network cyclical exchanges greeting message with the neighboring nodes, and maintains the adjacent node table. The table contains the following information: location of the node, adjacent node location, location of the sink node, the node $\mathrm{i}$ and sink point with the $\mathrm{X}$-axis formed by the angle $\left(\theta_{\mathrm{i}, \text { sink }}\right)$, the angle of the node and adjacent nodes $\left(\theta_{\mathrm{i}, \mathrm{j}}\right)$, and all need maintenance.

\section{B. The tree structure of data-aggregation based on the maximum lifetime}

Aggregation tree structure is the core of the algorithm. According to the established level of detection nodes, the node is actively looking for a route to the sink node, in the process of finding the shortest route as the main basis for routing, while taking into account to reduce the number of internal nodes of the tree structure, guarantee the transmission path to shorten the length of the tree structure under the premise of effective data-aggregation.

Data-aggregation tree formation process is as follows:

1) Initialization, all nodes in the detecting path are empty, all nodes of the father node and child node information are empty.

2) Select the father node: the detection node sends hello packets to trigger the operation of the node to find his father node. To send a message set current node $i$, $i$ will be in accordance with the node information table to select adjacent nodes the best next hop node. Set of adjacent nodes in the $\mathrm{Ni}$, i first select the minimum value of $\left|\theta_{\mathrm{i}, \text { sink }}-\theta_{\mathrm{i}, \mathrm{j}}\right|$ from $\mathrm{Ni}$ which is a set of node i's neighbor, look at the flag value of $\mathrm{j}$ :

(1) flag $=1$ node $\mathrm{i}$ directly set node $\mathrm{j}$ as its parent node, recording $\mathrm{Pi}=\mathrm{j}$, receiving hello message, in the collection of child nodes of $j$ to add nodes $i$, turn to step (3).

(2) flag $=0$ node $\mathrm{i}$ set node $\mathrm{j}$ as its father node, recording $\mathrm{Pi}=\mathrm{j}$, node $\mathrm{j}$ receives hello message, node $\mathrm{i}$ join the collection of child nodes $\mathrm{j}$, and reset flag $\mathrm{j}=1$, then go step (3). 


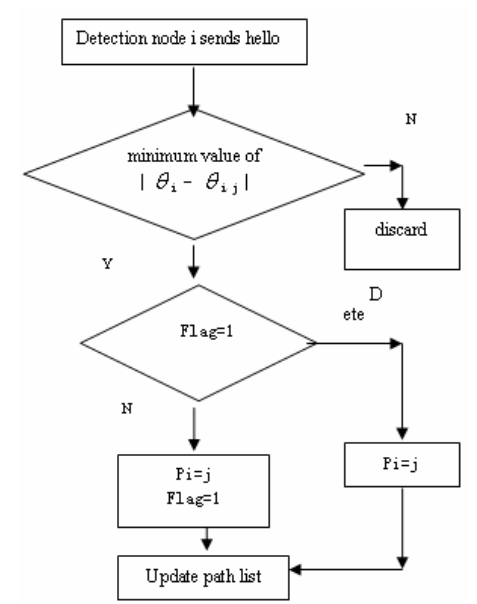

Figure 2. Node i's routing algorithm to find the parent node diagram

3) update path list: any node $\mathrm{j}$ in the network decided to receive lower nodes i send the HELLO packets, first extract the path list in the HELLO packet of information, i set the path list is $\mathrm{Li}=\{1,2, \ldots, \mathrm{j}\}$. Judgment the value of $\mathrm{Lj}$ :

a. $L j=$ NULL: set $L i=\{1,2, \ldots j\}, \quad$ directly add

$\mathrm{j}$ to the list, and send their updated information to neighbor nodes, turn (2).

b. $\operatorname{Lj} \neq$ NULL: Set $\mathrm{Lj}=\{\mathrm{j}, \mathrm{j}+1, \mathrm{j}+2, \ldots\}$, Let the information of $\mathrm{Lj}$ into the position after $\mathrm{j}$ in the $\mathrm{Li}$, updated $\mathrm{Li}=\{1,2, \ldots \mathrm{j}, \mathrm{j}+1, \mathrm{j}+2, \ldots\}$. Send to the neighbors to make their news updated, to (2).

4)Look over continuously updated Li, until it contains sink node.

When the algorithm ended, at the path end of all the detection nodes contains a sink node. Figure 2 is a node $i$ to find its father node routing diagram. Figure 3 is a dataaggregation tree formation algorithm diagram.

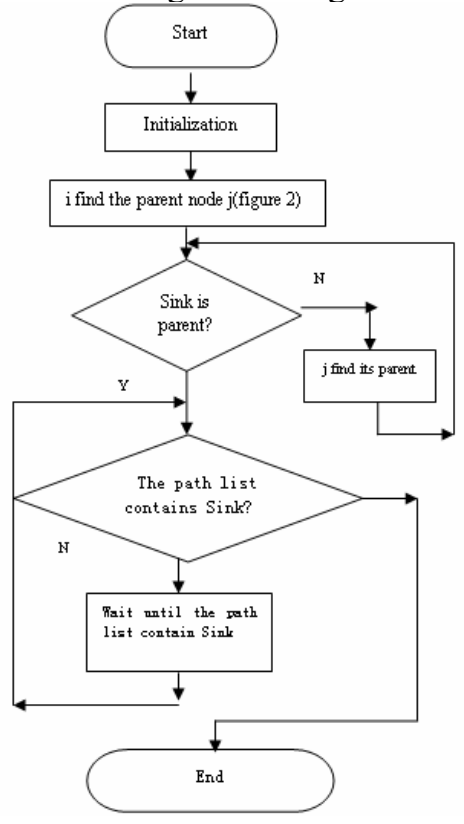

Figure 3. Diagram of data-aggregation tree formation algorithm

\section{Data reporting process}

As a final result, sink node is a root in the tree. In the tree structure, detection node reporting data detected to sink node is the main task of data reporting process. Tree nodes of type 1 can directly detect the data sent to the father node; type 2 nodes need to wait for the data from their child nodes and send it to its own parent after the data fusion is ready; type $=3$ node has child nodes which have to wait for the data reported in order to carry out data fusion, and reports the data to the parent node. Along the direction of data transmission through upper nodes detected, it reaches the sink node.

In this procedure, the main achievement is that the parent nodes process data sent by child node of lower layer with fusion, which requires the parent node to wait for the data of each child node to arrive, due to the length of different network paths or nodes encountered during data transmission congestion and other factors, the parent node can not receive the data and has been in a wait state, this case will reduce the efficiency of the network or lead to the loss of data. It is necessary that it has a reasonable waiting time for the fusion node to set the threshold [7]. When the wait time is exceeded, giving up the date which the child node has not arrived, the abandoned node number has been recorded for retransmission.

\section{AlgORITHM ANALYSIS}

The algorithm uses tree structure to achieve data fusion, finally, forms fusion tree structure. Its characteristic is better than random data fusion, avoided redundant data transmission, reduced the energy consumption of the whole network. Considered the principle of "shortest distance between two points is a straight line", select a node with the smallest deflection angle relatively to the sink node as a next hop, that is the path from the node to the sink in the shortest distance.

\section{CONCLUSION}

This paper proposed a date-aggregation algorithm based on maximum lifetime for wireless sensor networks, the algorithm based on determine the shortest route between each detection node and the sink node, based on the establishment of a data-aggregation tree. The composition of the tree structure make data-aggregation more obvious, thus saving energy; in the data transfer process, taking into account the remaining energy of nodes, timely adjustment of the structure of the tree, which makes the network's energy consumption to achieve a balanced, to avoid the path of individual node failure and cause of death retransmission. Simulation results show that the algorithm achieve better data integration, reducing the amount of data transmission, thereby saving energy and maximizing the network life cycle. 


\section{REFERENCES}

[1] I .F. Akyldiz, W .Su, Y. Sankarasubramaniam, E. Cayirci, Wireless sensor networks: a survey, Computer Networks 38(4)(March 2002) 393-422.

[2] Lin $\mathrm{k}$, Zhao $\mathrm{H}$ etc. Energy prediction and routing algorithm in wireless sensor networks[J]. Journal on Communications. 2006, 27(5):21-23.

[3] S.Madden, M.J.Franklin, J.M. Hellerstein, et al. TAG: a Tiny Aggregation Service for Ad-Hoc Sensor Networks. OSDI,2002

[4] Q.Gao,K.J.Blow,D.J.Holding et, Radio range adjustment for energy efficient wireless sensor networks[EB/OL]. www.elsevier.com/locate/adhoc,2006.

[5] L.Li and J.Y.Halpen, Minimum energy mobile wireless networks revisited.IEEE International Conference on Communications (ICC).2001.

[6] Manjeshwar A,Agarwal D P.TEEN: a routing protocol for enhanced efficiency in wireless sensor networks[C].1st Intl Wor-kshop on Parallel and Distributed Computing Issues in Wireless Networks and Mobile Computing, 2001.

[7] Heinzelman W R,Chandrakasan A, Balakrishnan H. Energy-efficient communication protocol for wireless microsensor networks[C]. Proc of the 33rd Intl Conf on System Sciences,2000:1-10. 